\title{
CÁNCER DE PENE. UNA REVISIÓN DE 18 CASOS
}

\author{
M. SOTO DELGADO, F. ARREDONDO MARTÍNEZ, G. PEDRERO MÁRQUEZ*, \\ B. BASQUURO GONZÁLEZ, A. ZURERA COSANO, R. LINARES ARMADA
}

Servicio de Urología. *Unidad de Enfermería. Hospital Infanta Elena. Huelva.

Actas Urol Esp. 27 (10): 797-802, 2003

\section{RESUMEN}

CÁNCER DE PENE. UNA REVISIÓN DE 18 CASOS

INTRODUCCIÓN: El carcinoma escamoso de pene es el tumor de pene más frecuente. Las metástasis ganglionares son relativamente frecuentes, pero la diseminación a distancia es muy rara.

MATERIAL Y MÉTODOS: Entre 1990 y el 2002 fueron diagnosticados y tratados 18 casos de carcinoma de pene. El seguimiento mínimo fue de 2 años de evolución (de 2 a 11 años, con una media de 49 meses).

El tratamiento de la lesión primaria, habitualmente por medio de la amputación parcial de pene, nos permite conocer el estadio patológico y el grado de diferenciación histológico, lo cual orientó primordialmente nuestra actitud frente a los ganglios linfáticos regionales.

RESULTADOS: Las adenopatías inguinales palpables persistieron tras antibioterapia en 6 de 7 pacientes. Se realizaron 4 linfadenectomías inguinales precoces. Las complicaciones post-operatorias se presentaron en los 4 casos, siendo el linfedema la más frecuente (100\%).

CONCLUSIONES: La categoría del $\mathrm{T}$ y el grado histológico de la lesión primaria al momento de decidir la conducta en el manejo de los ganglios, evitará linfadenectomías innecesarias y por otro lado tratará radical y oportunamente aquellos que presenten alto riesgo de diseminación ganglionar.

PALABRAS CLAVE: Pene. Carcinoma. Linfadenectomía.

\section{ABSTRACT}

CANCER OF THE PENIS. A REVIEW OF 18 CASES

INTRODUCTION: Squamous cell carcinoma is the most common tumor of the penis. Nodal metastases are relatively common, but distant disemination is very rare.

MATERIAL AND METHODS: From 1990 to 2002, we diagnosed and treated 18 cases of carcinoma of the penis. The minimum follow-up was 2 years (range 2-11 years; mean 49 months).

Treatment of the primary lesion is usually by partial amputation of the penis, which enables us to determine the pathological stage and the histological grade of the tumor and, consequently, our approach to the reguional lymph nodes.

RESULTS: Palpable inguinal nodes after antibiotherapy remained in six out of seven patients. Inguinal lymphadenectomy was performed early in 4 cases. Postoperative complications were present in the 4 cases, lymphedema being the most frequent one (100\%).

CONCLUSIONS: The $\mathrm{T}$ and the histological grade of the primary lesion must be considered when deciding the approach in the management of the lymph nodes as unnecesary lymphadenectomy can be avoided and those at high risk of lymph node invasion can be treated radically and timely.

KEY WORDS: Penis. Carcinoma. Lymphadenectomy. 
$\mathrm{E}^{1}$ carcinoma de pene es un tumor relativamente infrecuente en nuestro medio constituyendo menos del 1\% de las muertes por cáncer en el varón. En España, su tasa de prevalencia se sitúa en el $0,7 \%$ de los tumores malignos del varón ${ }^{1}$.

La máxima incidencia ocurre habitualmente en la sexta década de la vida y se trata de una enfermedad social relacionada con el subdesarrollo y clásicamente asociada con la higiene defectuosa y la exposición prolongada a irritantes o cancerígenos desconocidos del esmegma en individuos no circuncidados, infecciones virales, psoralenos y radiación ultravioleta ${ }^{1}$.

La lesión maligna más frecuente del pene es el carcinoma epidermoide y su variedad el carcinoma verrucoso ( $20 \%$ de todos los casos) $)^{1,2}$.

La actuación terapéutica en el carcinoma de pene incluye el tratamiento sobre la lesión primaria y la actitud activa o expectante sobre los ganglios linfáticos regionales (inguinales superficiales y profundos) ${ }^{3}$.

En este trabajo presentamos nuestra serie durante los últimos doce años (1990-2002) con 18 pacientes, que han podido ser seguidos clínicamente durante este periodo de tiempo.

\section{MATERIAL Y MÉTODO}

En un periodo de 12 años fueron tratados y controlados 18 pacientes con el diagnóstico de carcinoma de pene. El seguimiento de nuestros pacientes varía entre un mínimo de 2 años y un máximo de 11 años, con una media de 49 meses.

La edad de nuestros enfermos está comprendida entre los 43 y los 89 años con una media de 65,8 años. Los síntomas clínicos más frecuentes que motivaron la consulta fueron varios y destaca entre ellos la presencia de una lesión úlcerovegetante en el pene ( $50 \%$ de los casos), fimosis muy cerradas con supuración y/o sangrado y/o palpación de una tumoración prepucial $(38,8 \%$ de los casos), y masa inguinal (11,2\% de los casos). El tiempo de evolución del tumor primario hasta el momento del diagnóstico osciló entre 3 y 30 meses con un promedio de 11,8 meses.

La localización más frecuente de la lesión inicial fue el glande (50\%) seguida del prepucio $(22,2 \%)$, surco balanoprepucial $(16,7 \%)$ y cuerpo del pene $(11,1 \%)$.
Previa confirmación biópsica de la lesión, el tratamiento del tumor primario consistió en: penectomía parcial en 13 casos $(72,3 \%)$, penectomía total en 3 casos (16,6\%), exéresis de la lesión en 1 caso $(5,5 \%)$ y circuncisión en 1 caso $(5,5 \%)$.

Siete pacientes presentaron adenopatías inguinales palpables en el momento del diagnóstico de la lesión inicial. Estos pacientes fueron sometidos a un tratamiento antibiótico de amplio espectro durante seis semanas y posteriormente fueron reevaluados para establecer la necesidad o no de linfadenectomía inguinal. De los 7 pacientes, en tan sólo 1 enfermo desaparecieron las adenopatías inguinales tras la terapia antibiótica.

El estudio de extensión en todos los casos incluyó, además de la exploración física, la realización de un TAC abdominopélvico y una radiografía de tórax.

\section{RESULTADOS}

El tratamiento del tumor primario a través de las distintas técnicas utilizadas, permitió definir la estadificación patológica (siguiendo la clasificación TNM) y el grado de diferenciación celular de los tumores en los 18 pacientes (Tabla I).

\section{TABLA I}

RESULTADOS HISTOPATOLÓGICOS (pT Y GRADO DE DIFERENCIACIÓN CELULAR)

\begin{tabular}{|l|c|c|c|c|c|}
\hline & $p$ Tis & $p T a$ & $p T 1$ & $p T 2$ & $p T 3$ \\
\hline & 4 & 1 & & & \\
\hline G-I & & & 7 & & \\
\hline G-II & & & 1 & 1 & 3 \\
\hline G-III & & & & & 1 \\
\hline
\end{tabular}

El manejo de los ganglios linfáticos de los 18 pacientes de nuestra serie fue el siguiente:

- Seguimiento estricto en los 10 pacientes que no presentaban adenomegalias (NO) en el momento del diagnóstico de la lesión inicial y que no tenían indicación de linfadenectomía inguinal precoz según el estadio patológico y el grado de diferenciación celular del tumor primario.

- Seguimiento estricto en el paciente que presentaba adenopatías inguinales inicialmente y que desaparecieron tras tratamiento antibiótico de amplio espectro. 
- Linfadenectomía inguinal bilateral precoz en 1 paciente sin adenopatías palpables pero con un tumor primario pT3 GII.

- De los 6 pacientes que se presentaron con adenopatías persistentes $(\mathrm{N}+)$ posteriores a las 6 semanas de antibioterapia, en 3 se realizó linfadenectomía inguinal bilateral modificada y los otros tres casos se consideraron inoperables por presentar adenopatías inguinales fijas, adenopatías en cadenas ilíacas y metástasis a distancia $(\mathrm{M}+)$ tras realizar el estudio de extensión.

La estadificación patológica de los 4 pacientes sometidos a linfadenectomía inguinal fue pNO en 1 caso y pN1 (metástasis en un único ganglio inguinal superficial) en los tres casos restantes.

La relación entre la categoría $\mathrm{T}$ del tumor y la presencia de metástasis ganglionares palpables fue la siguiente: pT1 (2 casos), pT2 ( 1 caso), pT3 (3 casos). En lo referente a la diferenciación celular: G1 ( 1 caso), G2 (4 casos), G3 ( 1 caso).

El estadio patológico y el grado de diferenciación histológica de los 4 pacientes sometidos a linfadenectomía inguinal fue: pT3GII (pNO); pT1GII (pN1); pT1GI (pN1); pT2 GII (pN1).

A los 7 y 6 meses de la penectomía parcial (pT1GII) y de la linfadenectomía inguinal (pN1) respectivamente se objetivó en un 1 enfermo una recidiva tumoral en el muñón peneano lo cual obligó a realizar una penectomía total con uretrostomía perineal siendo el estadio patológico de la pieza quirúrgica pT3GII (en este momento el estudio de extensión fue negativo). A los 4 meses de la última cirugía, reaparecieron varias recidivas tumorales a nivel de la herida quirúrgica de la amputación peneana así como en la piel del hemiabdomen inferior y de ambos muslos (en el estudio de extensión practicado se objetivaban adenopatías ilíacas y periaórticas así como metástasis a distancia). El paciente falleció 2 meses más tarde.

En ninguno de los 11 pacientes sometidos a seguimiento estricto hemos encontrado recidiva tumoral a nivel inguinal durante el mismo.

En relación con las complicaciones post-operatorias de la linfadenectomía inguinal, destaca la infección local de la herida quirúrgica en 2 casos (2/4), el linfedema de miembros inferiores y edema escrotal en los 4 pacientes (100\%) sometidos a la disección de los ganglios inguinales.
De los 18 pacientes incluidos en el estudio, 8 $(44,4 \%)$ murieron durante el seguimiento. El 50\% $(4 / 8)$ murió por causas ajenas al tumor y el otro $50 \%(4 / 8)$ restante falleció como consecuencia del tumor de pene. En los 4 pacientes que murieron por causa tumoral, el estadio patológico de la pieza quirúrgica fue pT3 (100\% de los casos) y el grado de diferenciación celular fue G2 (3 casos) y G3 (1 caso).

La invasión tumoral de los ganglios linfáticos inguinales es un factor pronóstico de primera magnitud. Los 4 pacientes que fallecieron durante el seguimiento a causa del tumor peneano, presentaron adenopatías inguinales palpables en el momento del diagnóstico de la lesión inicial.

De los 3 pacientes sometidos a linfadenectomía inguinal con estadio patológico pN1, 1 enfermo falleció a los 13 meses de la amputación peneana parcial (ya comentado anteriormente) y los otros dos enfermos (pT2GII y pT3GII) sobreviven libres de enfermedad hasta el momento actual tras un seguimiento de 4 y 9 años respectivamente.

\section{DISCUSIÓN}

El carcinoma de pene es una enfermedad maligna poco frecuente en países desarrollados, 0,1-0,7 por 100.000 habitantes ${ }^{4}$. Sin embargo, en países subdesarrollados la incidencia se incrementa considerablemente hasta representar el 10-20\% de los tumores malignos en el varón. La fimosis y los procesos irritativos crónicos relacionados con una higiene defectuosa se asocian al desarrollo del cáncer de pene ${ }^{5}$. En nuestra serie, el 38,8\% de los casos presentaban antecedentes de fimosis, con frecuentes episodios de balanopostitis.

La edad habitual de este tipo de tumor se sitúa entre la $6^{\underline{a}}$ y la $7^{\underline{a}}$ década de la vida ${ }^{6,7}$, recogiéndose en nuestro estudio una edad media de 65,8 años. Al revisar la literatura, llama la atención la existencia de un prolongado retraso entre el momento de aparición de la lesión y la primera consulta diagnóstica ${ }^{1}$. En nuestros pacientes, el tiempo medio entre la aparición de la lesión y el momento de la primera consulta fue de 11,8 meses (similar al de otras series). El motivo de consulta más frecuente fue la presencia de lesión peneana (9 de 18 casos). 
El tumor primario se localiza preferentemente en glande en un $48 \%$, prepucio en el $21 \%$, en ambos el 9\%, en el surco balanoprepucial en el $6 \%$ y en el cuerpo peneano menos del $2 \%^{8}$. En nuestra serie, la localización más frecuente del tumor peneano fue el glande en un 50\% de los casos.

El diagnóstico de todas las lesiones de pene comienza por la toma de biopsia de las mismas (fundamentalmente cuando se sospecha lesión neoplásica) para confirmar su naturaleza tumo$\mathrm{ral}^{1}$. El diagnóstico histopatológico habitual es el carcinoma epidermoide en sus distintas formas de superficial, ulcerovegetante e infiltrante ${ }^{7,9}$. En nuestra serie, tan sólo un caso fue diagnosticado de otra forma histológica menos frecuente como el carcinoma verrucoso.

La calidad de vida es una demanda cada vez más frecuente por parte de los pacientes, lo que obliga a llevar a cabo terapias que dentro de la seguridad de no comprometer la vida del paciente permitan la conservación del órgano sexual. El tratamiento conservador del pene estaría indicado en tumores pequeños y no infiltrantes ${ }^{5}$. Entre las técnicas conservadoras disponemos de las siguientes:

- Cirugía conservadora: exéresis local de la lesión, circuncisión en casos circunscritos a prepucio y glandectomía en los limitados a glande ${ }^{10}$.

- Laserterapia. En la actualidad se considera la terapia conservadora de elección en el carcinoma de pene y su indicación más precisa es en tumores no infiltrantes menores de $2 \mathrm{~cm}^{11}$.

- Radioterapia externa o intersticial. Esta modalidad de tratamiento tiene unas indicaciones limitadas a tumores no infiltrantes menores de $4 \mathrm{~cm}$, aunque algunos Centros han abandonado estas técnicas por obtener unos resultados estéticos irregulares ${ }^{12}$.

En nuestra serie, tan sólo 2 pacientes eran subsidiarios de tratamiento conservador, realizándose exéresis de la lesión en un caso y una circuncisión en el caso restante.

La conservación peneana se empleará sólo si es posible asegurar un seguimiento estricto y prolongado, ya que no tenemos evidencia definitiva de las tasas de recidiva local y, por lo tanto, el diagnóstico de la misma debe ser precoz para que la supervivencia del paciente no se vea com- prometida $^{13}$. En ninguno de los 2 pacientes de nuestra serie sometidos a terapia conservadora se ha objetivado recidiva local tras un seguimiento medio de 76,5 meses.

En los tumores grandes de crecimiento superficial y en los infiltrantes, la cirugía clásica del carcinoma epidermoide de pene contempla la posibilidad de realizar una penectomía parcial o total. La mayor o menor radicalidad dependería de la consecución de $2 \mathrm{~cm}$ de margen tisular sano de la lesión junto con la obtención de una longitud peneana suficiente para dirigir el chorro miccional al orinar en posición de bipedestación. Si esto no se cree conseguible, se suele considerar como la mejor opción proceder a una penectomía total con uretrostomía perineal ${ }^{14}$. En nuestra serie, hemos realizado $13(13 / 18)$ penectomías parciales y $3(3 / 18)$ penectomías totales.

Ya que la complicación más frecuente de la penectomía es la aparición de una estenosis del neomeato peneano o a nivel de la uretrostomía perineal, lo cual ocurre en el $6 \%$ de los casos, la técnica debe incluir la espatulación de la uretra ${ }^{7}$. De las 16 penectomías realizadas en nuestros pacientes, tan sólo hemos tenido un caso con estenosis de neomeato $(6,25 \%)$.

Tras la amputación peneana, la recurrencia local es rara cuando se respetan unos márgenes adecuados (2-6\%) pero puede aparecer ocasionalmente obligando a una segunda intervención ${ }^{15}$. En nuestro trabajo, se objetivó una recurrencia local tras penectomía parcial lo que obligó a realizar posteriormente una penectomía total.

El proceso metastásico del cáncer de pene se desarrolla esencialmente por etapas, en una primera el tumor afecta a los ganglios inguinales, posteriormente las cadenas iliacas $\mathrm{y}$, finalmente, se desarrollan las metástasis a distancia (menos del $10 \%$ de los casos) ${ }^{5}$. En el momento del diagnóstico de la lesión inicial alrededor del 50\% de los pacientes presentan adenopatías inguinales palpables, de ellas sólo cerca de la mitad serán tumorales ya que con frecuencia el cáncer de pene suele estar infectado y provocar adenomegalias inflamatorias. Por los motivos infecciosos citados debe plantearse, en los pacientes con adenopatías inguinales palpables, la antibioterapia de amplio espectro durante 6 semanas antes de indicar cualquier actuación regional $^{1}$. Como alternativa al tratamiento antibió- 
tico, se propone la biopsia percutánea de las adenopatías para confirmar la naturaleza neoplásica de las mismas ${ }^{5}$. En nuestra serie, siete pacientes presentaron adenopatías inguinales palpables en el momento del diagnóstico de la lesión inicial, y tras tratamiento antibiótico sólo desaparecieron las adenomegalias en 1 paciente.

Mención especial merece los 3 casos de nuestra serie que presentaban metástasis a distancia en el momento del diagnóstico inicial.

En los pacientes con adenopatías palpables tras tratamiento antibiótico o después de confirmar su naturaleza neoplásica mediante biopsia percutánea, la indicación de linfadenectomía inguinal bilateral no es cuestionada ya que la supervivencia de los pacientes tratados con linfadenectomía es del $50 \%$, lo que indica un gran valor terapéutico ${ }^{1,3,5,6,16}$. De los 6 pacientes de nuestra serie con ganglios inguinales persistentes tras tratamiento antibiótico, 3 fueron sometidos a linfadenectomía inguinal bilateral mientras que los otros 3 casos restantes se consideraron inoperables por presentar enfermedad diseminada.

De los pacientes sin adenopatías palpables inguinales, el $17 \%$ albergan micrometástasis en una o en ambas cadenas ${ }^{17}$. En estos pacientes se han identificado grupos de riesgo para optimizar la indicación de linfadenectomía inguinal profiláctica $^{18}$. En general, se puede considerar:

- El grupo de bajo riesgo incluiría a aquellos pacientes con tumores TlG1. La indicación en este grupo de pacientes es la vigilancia estricta que consistiría en exploración física cada 2 meses durante dos años y después cada 6 meses ${ }^{19}$.

- El grupo de alto riesgo comprendería a pacientes con altos estadios (T2-4) asociado a grado moderado (G2) y a aquellos con tumores pobremente diferenciados (G3). En estos pacientes estaría indicada la linfadenectomía inguinal bilateral modificada ${ }^{20}$. A un paciente de nuestra serie se le practicó la linfadenectomía inguinal profiláctica por presentar un tumor primario pT3 GII.

- El grupo intermedio incluye a pacientes con tumores T1G2 o T2G1. En estos casos se tendrían en cuenta otros factores de predicción como la invasión vascular o linfática, el patrón de crecimiento o circunstancias personales relacionadas como la elección personal, nivel cultural, posibilidad de cumplir un programa de seguimiento, etc.
Si en aquellos pacientes sometidos a un programa de vigilancia aparece una adenopatía tiempo después del tratamiento del tumor primario, se debe realizar una linfadenectomía unilateral ya que las metástasis ganglionares aumentarán de tamaño a la misma velocidad, y si estuvieran presentes en ambas ingles, deberían aparecer al mismo tiempo5,16,17. En ninguno de nuestros pacientes sometidos a seguimiento estrecho han aparecido adenopatías durante el mismo.

La morbilidad de la linfadenectomía inguinal es elevada (30-50\%) estando relacionada con problemas de edemas en miembros inferiores y, en menor medida, edemas peneo-escrotales, además de cicatrices antiestéticas en áreas inguinales por necrosis cutáneas en el post-operatorio inmediato $^{3}$. Las complicaciones halladas en nuestra casuística son similares a las comentadas en la literatura con una incidencia de linfedema del $100 \%$.

Según la literatura, la linfadenectomía pélvica estaría indicada en pacientes con 2 o más adenopatías inguinales positivas ${ }^{5}$. En nuestra serie no hubo indicación de linfadenectomía pélvica en ningún caso.

\section{CONCLUSIONES}

La relativa escasa frecuencia del carcinoma de pene en nuestro medio, ha hecho que despierte menos atención que otros tumores genitourinarios.

En los pacientes considerados de bajo riesgo, la vigilancia estricta es una opción de tratamiento real.

En los pacientes considerados de alto riesgo, no debe dudarse en practicar la linfadenectomía inguinal bilateral de manera precoz ya que la misma tiene un claro efecto sobre la supervivencia.

En el grupo de pacientes de riesgo intermedio, se tendrán en cuenta otra serie de factores para decidir la actitud más óptima.

\section{REFERENCIAS}

1. VIRSEDA JA, SALINAS A, HERNÁNDEZ I.: Carcinoma de pene. ¿Qué hacer con los ganglios linfáticos regionales?. Arch Esp Urol 1994; 47: 349-362.

2. AROCENA F.: Carcinoma de pene. Editorial. Arch Esp Urol 1989; 42: 293. 
3. RUBIO-BRIONES J, VILLAVICENCIO H, REGALADO R et al.: Carcinoma escamoso de pene; protocolo de tratamiento según nuestra experiencia en 14 años. Arch Esp Urol 1997; 50: 473-480.

4. PERSKY L.: Epidemiology of cancer of the penis. Recent Results Cancer Res 1977; 60: 97.

5. SOLSONA E.: Cáncer de pene. Actas Urol Esp 2002; 26: 525-531.

6. CÓZAR JM, NAVARRO J, DE LA PEÑA J et al.: Controversias sobre la linfadenectomía regional en el carcinoma de pene. Arch Esp Urol 1991; 44: 951955.

7. PIZZOCARO G, PIVA L, BENDIERAMONTE G, TANA S.: Up-to-date management of carcinoma of the penis. Eur Urol 1997; 32: 5-15.

8. SUGRIN G, HUBEN R.: Bening and malignant lesions of the penis. In: Adult and Pediatric Urology. 2 $2^{\mathrm{a}}$ ed. Gillenmater JY (ed). Year Book Medical Published. Chicago 1991; 1643.

9. ADEYOJU AB, THORNHILL J, CORR J, GRAINGER R, MCDERMOTT TED, BUTLER M.: Prognostic factors in squamous cell carcinoma of the penis and implications for management. Br J Urol 1997; 80: 937-939.

10. BISSADA NK.: Conservative extirpative treatment of cancer of the penis. Urol Clin North Am 1992; 19: 283-290.

11. HORENBLAS S, VAN TITEREN H, DELAMERRE JF, MOONEN LM, LUSTIG V, VAN WAARDENBURG EW.: Squamous cell carcinoma of the penis. II. Treatment of the primary tumor. J Urol 1992; 147: 1533-1538.

12. CROOK J, GRIMARD L, TSIHHLIAS J, MORASH C, PANZARELLA T.: Interstitial brachyterapy for penile cancer: an alternative to amputation. $J$ Urol 2002; 167: 506-511.

13. KOCH MO, SMITH JA.: Local recurrence of squamous cell carcinoma of the penis. Urol Clin N Amer 1994; 21: 739 .
14. KLEIN EA.: Partial and total penectomy for cancer. Urol Clin North Am 1991; 18: 161-169.

15. DERAKHSHANI P, NEUBAUER S, BRAUN M, BARGMAN H, HEIDENREICH A, ENGELMANN U.: Results and 10-year follow-up in patients with squamous cell carcinoma of the penis. Urol Int 1999; 62: 238-244.

16. CRUZ NA, ALLONA A, CLEMENTE L, LINARES A, BRIONES G, ESCUDERO A.: Linfadenectomía en el carcinoma escamoso de pene: revisión de nuestra serie. Actas Esp Urol 2000; 24: 709-714.

17. HORENBLAS S, VAN TITEREN H, DELAMERRE JF, MOONEN LM, LUSTIG V, VAN WAARDENBURG EW.: Squamous cell carcinoma of the penis. III. Treatment of regional lymph nodes. J Urol 1993; 149: 492-497.

18. SOLSONA E, IBORRA I, RUBIO J et al.: Prospective validation of association of local stage and clinically negative inguinal lymph-nodes as predictive factor for ocult lymph node micrometastases in patients with penile carcinoma. J Urol 2001; 165: 1506.

19. LYNCH DF, SCHELLHAMMER PF.: Conceptos contemporáneos de la linfadenectomía ilioinguinal del carcinoma escamoso de pene. AUA Update Series (edición española) 1998; 4: 15-21.

20. PARRA RO.: Accurate staging of carcinoma of the penis in men with nonpalapble inguinal lymph nodes by modified inguinal lymphadenectomy. $J$ Urol 1996; 155: 560-563.

Dr. M. Soto Delgado

C/ San Salvador, $6-3^{\circ}$ A

21003 Huelva

(Trabajo recibido el 25 julio de 2003) 